\title{
Low-temperature Near-field Scanning Optical Microscope for UV-visible Spectroscopy of Nanostructures
}

\author{
Sang-Youp Yim, Jong Su Kim, Yong Hwan Kim, Clare C. Byeon, Ok-Hwan Cha and Mun Seok Jeong* \\ Nanophotonics Laboratory, Advanced Photonics Research Institute, \\ Gwangju Institute of Science and Technology, Gwangju 500-712 \\ D. J. PARK, S. B. ChOI and D. S. Kim \\ Center for Subwavelength Optics and Department of Physics and Astronomy, Seoul National University, Seoul 151-747 \\ Ch. LiEnAU \\ Carl von Ossietzky Universität Oldenburg, Institut für Physik, 26111 Oldenburg, Germany
}

(Received 20 January 2010)

\begin{abstract}
A low-temperature near-field scanning optical microscope (LT-NSOM) is designed and realized in order to study the optical properties of nanostructures at cryogenic temperatures in the UV-visible wavelength range. Due to the simple and extensive optical design, any type of NSOM operation mode is accessible. In particular, the localized photoluminescence spectra of nanostructures can be measured at temperatures from $13 \mathrm{~K}$ up to $300 \mathrm{~K}$. We successfully demonstrate the performance of our LT-NSOM by investigating InGaN/GaN multiple quantum wells grown on a sapphire substrate at $15 \mathrm{~K}$.
\end{abstract}

PACS numbers: 07.79.Fc, 07.20.Mc, 07.60.Rd, 78.55, 78.66

Keywords: Near-field scanning optical microscopy, Cryogenic instrumentation, UV spectroscopy, Photoluminescence, Nanostructures

DOI: $10.3938 / \mathrm{jkps} .56 .717$

\section{INTRODUCTION}

The near-field scanning optical microscope is one of the most powerful systems used in the optical characterization of nanostructures, such as semiconductor nanostructures, single molecules, metallic particles, nano-slit arrays, electro-optic devices, and biological samples [16]. Unsurprisingly, many variants of NSOM exist for different experimental environments. One of the useful modifications is to add a low-temperature capability, cooling samples down to few $\mathrm{K}$, in order to increase the luminescence quantum yield and suppress the homogeneous linewidth broadening due to thermal excitation. The low-temperature NSOM systems have been successfully applied to quantum wells, quantum wires, magnetic heterostructures, and single quantum dot spectroscopy [7-11].

However, almost all of the LT-NSOM systems currently being used cool down the entire system, including the near-field probe, shear-force sensor, piezo scan stage, and even microscope objective lens. Therefore, severe drawbacks are unavoidable [7]. First, several hours

*E-mail: mjeong@gist.ac.kr, dsk@phya.snu.ac.kr are required to cool the large volume of the entire system to reach the thermal equilibrium state. Second, the scan range of the piezo scanner at $4 \mathrm{~K}$ is reduced to about $20 \%$ of its room temperature value due to inherent thermal characteristics of piezoceramic materials. Last, the shear-force detection conditions change continually with temperature. In order to circumvent the problems, Behme et al. designed and constructed a vacuum NSOM. In that design, they attached the sample to the cold finger of a helium flow cryostat to control the temperature of the sample in the range between $8 \mathrm{~K}$ and $330 \mathrm{~K}$ while keeping all the components of the NSOM close to room temperature by thermally isolating them from the sample in a vacuum chamber [12]. Therefore, they realized a wide scan range, as well as rapid cooling. Similarly, Gray and $\mathrm{Hsu}$ reported a variable temperature NSOM design operating over a temperature range of $12-300 \mathrm{~K}$ [13]. They used a copper braid as a thermal link between the sample stage and the cryostat cold finger.

In this paper, we report on the design and operation of a versatile LT-NSOM for UV-visible spectroscopy of nanostructures. In order to scan the sample, we connect the sample holder to the cryostat cold finger by using a flexible gold braid, similar to a previous work [13]. The sample holder is attached to a piezo scan stage with 
a glass plate between them. In general, sample scanning is preferable to tip scanning because the tip is fixed relative to the collection optics [14]. Consequently, our LT-NSOM system can operate in any type of configuration, such as illumination, collection or reflection mode. In particular, we put special emphasis on the UV-visible spectroscopy capability. We will demonstrate the performance of our system for photoluminescence (PL) mapping, by UV laser excitation, of InGaN/GaN multiple quantum wells (MQWs) grown on a sapphire substrate.

\section{LT-NSOM DESIGN}

A schematic diagram of the LT-NSOM scan head is shown in Fig. 1. A cylindrical vacuum chamber (56$\mathrm{cm}$ diameter, 30-cm height) is used to keep the NSOM scan head at a pressure below $10^{-5}$ mbar, which assures thermal isolation from the external environment. The chamber has four quartz windows for optical beam transmission and monitoring, four electrical connection ports for instrument control, and a hole for a near-field optical fiber probe. The quartz window has a transmittance of $\geq 0.9$ for wavelengths from $250 \mathrm{~nm}$ to $2 \mu \mathrm{m}$. In order to get high vacuum pressure, we use a rotary pump and a magnetic bearing turbo-molecular pump (STP451, BOC Edwards Inc.). In particular, the mechanical vibration of the turbo-molecular pump is less than $10 \mathrm{~nm}$ nominally, so the vibration is negligible even for tip scanning. Therefore, it is possible to keep the pump on throughout the experiments, keeping a high vacuum environment.

As stated above, thermal isolation of the NSOM scan head from the sample can prevent a reduction of scan range, and can keep the resonance condition of the shearforce sensor nearly the same for various temperatures [12]. In order to cool down the sample, we used a flexible gold braid to connect the sample holder and the cold finger of a continuous flow cryostat for ultra-highvacuum environment (Janis, ST-400), which has nominal cryogen consumption rate of $500 \mathrm{ml}$ per hour at $5 \mathrm{~K}$. The lowest temperature of the sample holder is $13 \mathrm{~K}$ when liquid $\mathrm{He}$ is supplied and $90 \mathrm{~K}$ when liquid $\mathrm{N}_{2}$ is supplied. We monitor and control the temperature of the sample holder to range from $13 \mathrm{~K}$ to $300 \mathrm{~K}$ by using a temperature controller (Neocera, LTC-11) with a temperature stability of less than $0.1 \mathrm{~K}$.

To avoid thermal conduction, we insert a glass plate between the sample holder and the piezo scan stage. The stage is a vacuum-compatible version (Physik Instrumente, P-620.2VL), which is linearized by closed loop operation using capacitive sensors. The maximum scan range is $50 \mu \mathrm{m}$ for each axis. In addition, we use two $x$ - $y$ $z$ linear translation stages driven by motorized actuators for independent coarse positioning of the sample and the probe. Therefore, we can remotely switch samples on the sample stage without breaking the vacuum condition or raising the temperature of the sample stage.

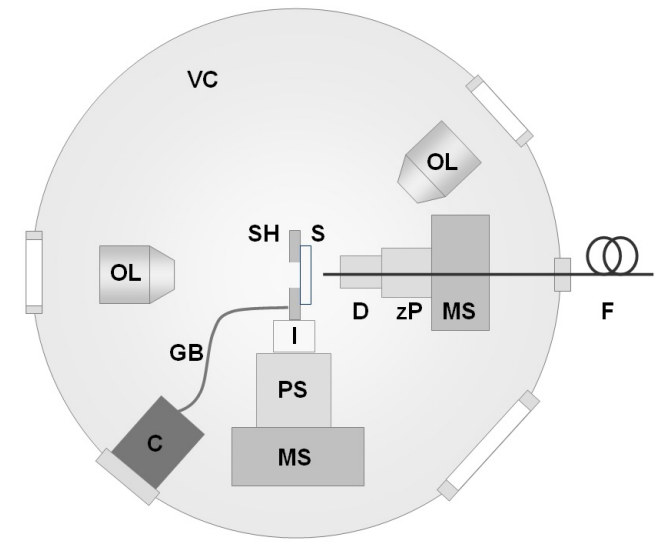

Fig. 1. Schematic diagram of the NSOM scan head in a vacuum chamber (top view). Shown are the (C) cold finger, (D) dither piezo, (F) fiber probe, (GB) gold braid, (I) insulator, (MS) motorized stage, (OL) objective lens, (PS) piezo scan stage $(\mathrm{x}-\mathrm{y}),(\mathrm{S})$ sample, $(\mathrm{SH})$ sample holder, $(\mathrm{VC})$ vacuum chamber, and $(\mathrm{zP}) \mathrm{z}$ piezo. In addition, three optical view ports are shown, which are used for this experiment.

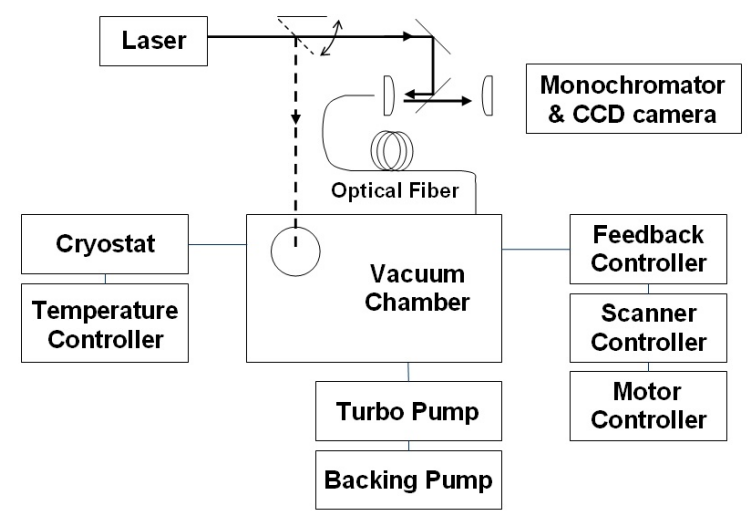

Fig. 2. Block diagram of the LT-NSOM setup. Using a flip mirror, the laser beam can reach the sample either through a focusing lens after passing the transmission window or through a NSOM optical fiber probe by launching optics. In the latter mode, the same fiber probe is used to collect the PL signal.

As shown in Fig. 1, our optical setup is simple and easy to extend, compared to that of the conventional upright standing design, which requires some vertical alignment. As the scan data show (Fig. 4), mechanical instability is negligible. For transparent samples, we place an objective lens behind the sample holder to collect the transmitted light. Otherwise, an objective lens with a long working distance $(17 \mathrm{~mm}, 50 \mathrm{X}$ Mitutoyo Plan NIR, 0.42 N.A.) is placed at an angle of 45 (with respect to the optical axis to collect the signal in reflection mode. The same objective lens, combined with a charge coupled device (CCD) camera outside the chamber, is used to monitor the sample and the probe.

Figure 2 is a block diagram of the LT-NSOM setup. We use an 8-mW, 375-nm UV laser diode (Oplink Com- 


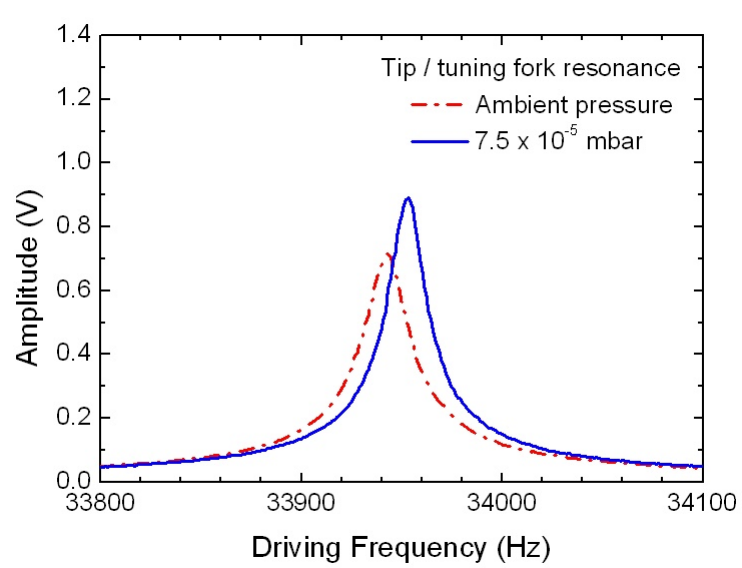

Fig. 3. Typical frequency response of shear-force sensor at ambient and vacuum pressures.

munications Inc.) as an excitation source. For the illumination mode, the excitation laser beam is launched to one end of NSOM fiber probe outside the vacuum chamber. The fiber passes through a hole in the vacuum chamber and we subsequently fill the hole. The NSOM fiber probe made of UV-grade fused silica is a commercially available $100-\mathrm{nm}$ aperture probe coated with $\mathrm{Al}$ or $\mathrm{Au}$ on the tapered fiber tip. If a flip mirror is used, the excitation laser beam can also be directly delivered to the sample through the quartz window, and the fiber probe collects the PL signal from the sample. For UV light excitation, this mode is preferred to the illumination mode due to the severe loss. After passing a dichroic beamsplitter and a long pass filter, the signal is dispersed by a monochromator with a 30-cm focal length (SP300i, Acton Research Corp.) and is detected by a thermoelectric cooled CCD camera (Pixis 100, Princeton Instruments).

A quartz tuning fork is used for shear-force sensing [15]. The NSOM fiber probe/tuning fork assembly is excited externally by a dither piezo. Figure 3 shows a typical frequency response of the shear-force sensor. The quality factor is about 1000 at ambient pressure, but increases slightly to 1200 with decreasing pressure. The resonance signal is monitored and fed to an analog feedback controller to regulate the distance between the sample and the tip as $\sim 10 \mathrm{~nm}$.

\section{RESULTS AND DISCUSSION}

We examine the scanning performance of the system by measuring the surface topography of a patterned InGaN film at $300 \mathrm{~K}$ and $15 \mathrm{~K}$. The recorded shear-force topography images taken at $300 \mathrm{~K}$ and $15 \mathrm{~K}$ are shown in Figs. 4(a), and 4(c), respectively. For comparison at two different temperatures, the dotted box region of Fig. 4(a) is magnified in Fig. 4(b). As shown in the figure, due to thermal isolation of the NSOM scan head from
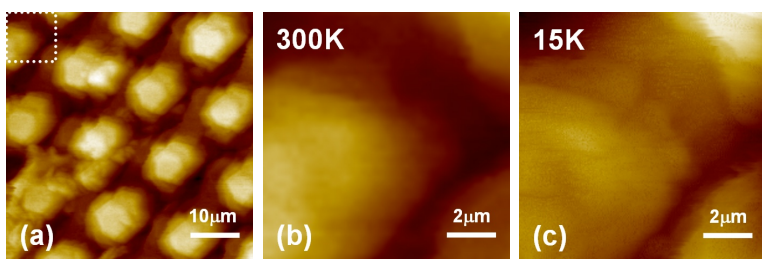

Fig. 4. (a) Surface topography image of a patterned InGaN film at $300 \mathrm{~K}$. (b) Magnified image of the dotted box region in (a). (c) Corresponding image at $15 \mathrm{~K}$.

the cold sample, no noticeable change of scan range can be found.

In order to demonstrate the performance of the LTNSOM setup, we measure the near-field PL of InGaN/GaN MQWs grown on a sapphire substrate [16]. The InGaN/GaN MQW sample was grown by using low-pressure metal-organic chemical vapor deposition (MOCVD) in a horizontal-type reactor. We used trimethylgallium $\left(\mathrm{Ga}\left(\mathrm{CH}_{3}\right)_{3}\right)$, trimethylindium $\left(\mathrm{In}\left(\mathrm{CH}_{3}\right)_{3}\right)$, and ammonia $\left(\mathrm{NH}_{3}\right)$ as the $\mathrm{Ga}$, In, and $\mathrm{N}$ precursors, respectively. First, a $2-\mu \mathrm{m}$ undoped GaN buffer layer was grown on a sapphire substrate at $1120^{\circ} \mathrm{C}$. Subsequently, 7 periods of InGaN/GaN MQWs were grown on the GaN buffer at $770{ }^{\circ} \mathrm{C}$. The MQWs consist of a $2-\mathrm{nm}$ InGaN well with an In content of about $25 \%$ and a 11-nm GaN barrier. Finally, a 200-nm GaN capping layer was grown at $1120^{\circ} \mathrm{C}$.

InGaN/GaN MQWs are well known to suffer a compressive biaxial strain due to the large lattice mismatch between the InGaN and the GaN layers. The strain may be relaxed via the formation of quantum-dot (QD)-like structures [17]. The strain-induced In composition fluctuation is the main factor responsible for the clustering and the distribution inhomogeneity of the dot-like region of the InGaN/GaN MQWs grown on the sapphire substrate.

A $5 \times 5 \mu \mathrm{m}^{2}$ near-field PL image of InGaN/GaN MQWs grown on a sapphire substrate was taken in the collection mode at a sample temperature of $15 \mathrm{~K}$ by using UV laser excitation (Fig. 5(a)). Similar to the room-temperature results, island-like bright spots are shown, but the bright spots are generally smaller than those in the room-temperature near-field PL image [16]. The cross sections of the bright spots exhibit a size of $400-500 \mathrm{~nm}$ full width at half maximum (FWHM). In addition, most of the acquired PL spectra are narrow bands of $20 \mathrm{~nm}$ (FWHM). The dotted bright spots A, $\mathrm{B}$, and $\mathrm{C}$ in Fig. 5(a) have 528-nm, 536-nm, and 533-nm peak wavelengths, respectively (Fig. 5(b)). We strongly believed that the QD-like islands show more significant localization due to the carrier confinement effect at low temperature. 


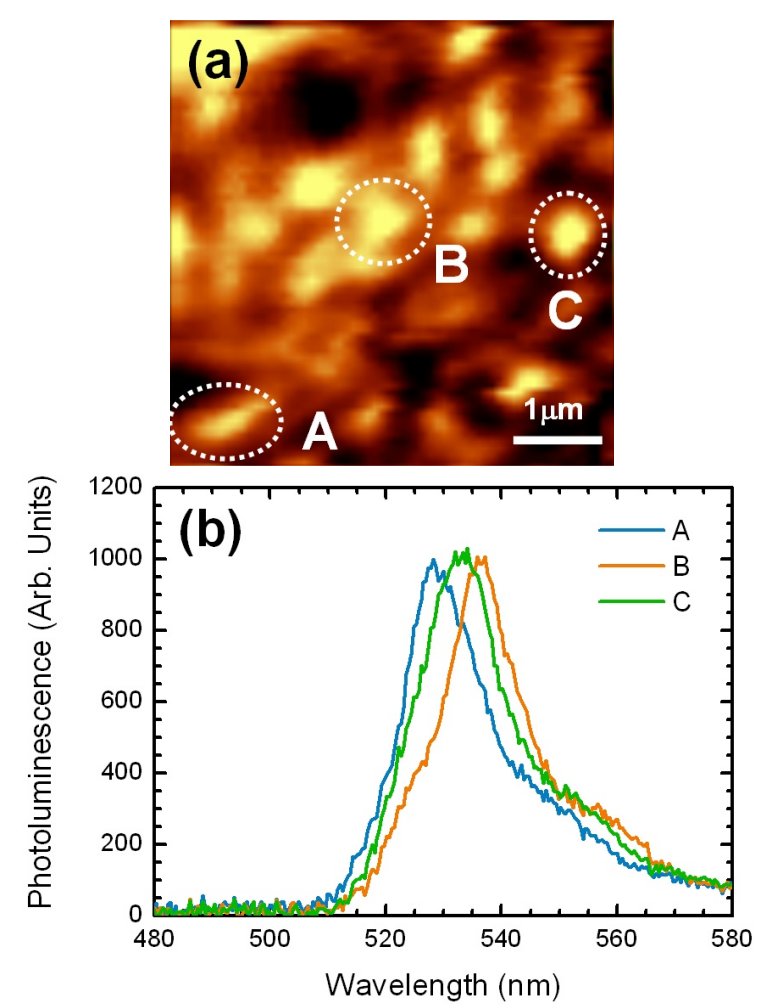

Fig. 5. (a) Near-field PL image and (b) the corresponding spectra, obtained at $15 \mathrm{~K}$ by using UV laser excitation $(5 \times 5$ $\mu \mathrm{m}^{2}$ ), of InGaN/GaN MQWs grown on a sapphire substrate.

\section{CONCLUSION}

In summary, we have designed and realized a lowtemperature near-field scanning optical microscope (LTNSOM) to study the optical properties of nanostructures at cryogenic temperatures in the UV-visible wavelength range. Due to the simple and extensive optical design, any type of NSOM operation mode is easily selectable and accessible in our LT-NSOM. In particular, the localized PL spectra of nanostructures can be measured at temperatures from $13 \mathrm{~K}$ up to $300 \mathrm{~K}$. We have successfully demonstrated with fairly good spatial resolution the acquisition of near-field PL images and the corresponding spectra of InGaN/GaN MQWs grown on a sapphire substrate at $15 \mathrm{~K}$ by using UV laser excitation. In general, the island-like bright spots are smaller compared to those of room-temperature measurements. We strongly believe that the QD-like islands show more significant localization due to carrier confinement effect at low temperatures.

\section{ACKNOWLEDGMENTS}

This work was supported by the Nano R\&D Program (2007-02939) through the National Research Foundation of Korea (NRF) funded by the Ministry of Education, Science and Technology (MEST).

\section{REFERENCES}

[1] H. F. Hess, E. Betzig, T. D. Harris, L. N. Pfeiffer and K. W. West, Science 264, 1740 (1994).

[2] W. E. Moerner and M. Orrit, Science 283, 1670 (1999).

[3] T. Klar, M. Perner, S. Grosse, G. von Plessen, W. Spirkl and J. Feldmann, Phys. Rev. Lett. 80, 4249 (1998).

[4] S. B. Choi, D. J. Park, D. S. Kim, M. S. Jeong and C. C. Byeon, J. Korean Phys. Soc. 53, 713 (2008).

[5] M. F. García-Parajó, B. I. de Bakker, M. Koopman, A. Cambi, F. de Lange, C. G. Figdor and N. F. van Hulst, NanoBiotechnology 1, 113 (2005).

[6] M. S. Jeong, D-K. Ko, J. Lee, H. J. Kim, J. Kim, Y-W. Kim, E-K. Suh and J. O. White, J. Korean Phys. Soc. 47, S209 (2005).

[7] S. I. Jung, H. Y. Yeo, I. Yun, I. K. Han, S. M. Cho and J. I. Lee, J. Korean Phys. Soc. 50, 763 (2007).

[8] R. D. Grober, T. D. Harris, J. K. Trautman and E. Betzig, Rev. Sci. Instrum. 65, 626 (1994).

[9] J. Levy, V. Nikitin, J. M. Kikkawa, D. D. Awschalom and N. Samarth, J. Appl. Phys. 79, 6095 (1996).

[10] S-K. Eah, W. Jhe and Y. Arakawa, Appl. Phys. Lett. 80, 2779 (2002).

[11] K. Matsuda, T. Saiki, S. Nomura, M. Mihara, Y. Aoyagi, S. Nair and T. Takagahara, Phys. Rev. Lett. 91, 177401 (2003).

[12] G. Behme, A. Richter, M. Süptitz and Ch. Lienau, Rev. Sci. Instrum. 68, 3458 (1997).

[13] M. H. Gray and J. W. P. Hsu, Rev. Sci. Instrum. 70, 3355 (1999)

[14] M. A. Paesler and P. J. Moyer, Near-field Optics (Wiley, New York, 1996), p. 114.

[15] K. Karrai and R. D. Grober, Appl. Phys. Lett. 66, 1842 (1995).

[16] T. V. Cuong, J. Y. Park, M. S. Kumar, C-H. Hong, E. K. Suh and M. S. Jeong, Jpn. J. Appl. Phys. 46, L372 (2007).

[17] D. I. Florescu, S. M. Ting, J. C. Ramer, D. S. Lee, V. N. Merai, A. Parkeh, D. Lu and E. A. Armour, Appl. Phys. Lett. 83, 33 (2003). 